\title{
Concrete Pavement Service Condition Assessment Using Infrared Thermography
}

\author{
Yang Lu, Aidin J. Golrokh, and MD Aminul Islam \\ Boise State University, 1910 University Drive, Boise, ID 83725-2060, USA \\ Correspondence should be addressed to Yang Lu; yanglufrank@boisestate.edu
}

Received 15 April 2017; Accepted 21 June 2017; Published 26 July 2017

Academic Editor: Hainian Wang

Copyright (C) 2017 Yang Lu et al. This is an open access article distributed under the Creative Commons Attribution License, which permits unrestricted use, distribution, and reproduction in any medium, provided the original work is properly cited.

\begin{abstract}
Infrared thermography (IRT), an effective nondestructive testing method, is used to obtain an initial evaluation of the concrete pavement surface and near surface in a time effective manner. In this paper, the effect of the depth of delamination inside concrete pavement on infrared thermography technique is studied for bridge decks inspection. To be able to mimic the delamination in subsurface, two Styrofoam cubes have been inserted in different depth near the surface of the concrete cylinder. After heating up the specimen, thermal images were taken from the surface using an infrared thermal camera to evaluate the effect of subsurface defects on detection sensitivity and accuracy. We also investigated the precision to which the shape and the size of the subsurface anomalies can be perceived using an uncooled thermal camera. To achieve this goal, we used image processing technique to accurately compute the size of delamination in order to compare it with the actual size. In addition, distance/thermal graph is used to detect the presence of the defect underneath the concrete surface. Furthermore, thermal transfer modeling was adopted in this paper to assist the setup of this experiment and the results are compared with laboratory findings.
\end{abstract}

\section{Introduction}

Infrared thermography (IRT) is a fast and easy to use nondestructive examination method that enables us to detect near surface delamination in a wider spectrum and with an acceptable accuracy compared to visual testing method [1]. IR inspection does not need direct contact with the surface of the bridge. IR imaging data can be collected using a camera that is installed on top of a vehicle that can travel with a speed of $16 \mathrm{~km} / \mathrm{h}(10 \mathrm{mph})$ [2]. These advantages give the inspector the opportunity to manage the inspection process on the bridge without interfering with the traffic [3]

IRT captures the temperature gradient of concrete pavement surfaces. IR camera is going to be able to capture clear temperature gradients on the concrete pavement surface at certain time windows during the heating or cooling cycles [4]. When exposed to heat, delaminated parts of concrete will interrupt the heat transfer since they have a lower thermal conductivity compared to concrete; therefore spots that are located on top of the delamination will be shown as hotter than the corresponding spots on top of sound parts [5]. This is actually the case when ambient heat is applied on top surface of the specimen or structure. The heat source can be either natural, such as the sun, or artificial, such as the high-power ultrasound [6]. We should note that in the case of internal heating or applying heat from underneath the specimen, a normal practice in laboratory experiments, the surface that is exposed to the delamination will appear cooler than the sound area in a thermal image. The reason for that is the same as is mentioned earlier.

Passive and active thermal imagery are the two types of IR inspection methods [7]. In passive approach, inspection is conducted without using artificial heating while in active approach, specimen is heated up artificially and then the temperature gradient is measured during heating or cooling cycles [8]. One very popular type of active thermography is Pulsed Thermography (PT). In this method, the depth of the delamination can be investigated by recoding the temperature decay of the specimen. After deploying heat to the specimen, the surface temperature will be recorded using a temporal plotting that gives us the temperature with respect to relative time (seconds). There will be a rapid temperature change on the surface due to thermal propagation by diffusion and radiation and convection losses. Delamination 
will affect the rate of diffusion; therefore, the delaminated area appears to have a different temperature when inspected using live thermal imagery. Deeper defects will have a later observation rate compared to the shallower ones. This is because the observation time $(t)$ and the depth $(z)$ are correlated [9]; that is, $t$ is in fact the square of $z$ and the loss of contrast $(c)$ is proportional to the cube of the depth [10]:

$$
\begin{aligned}
& t \approx \frac{z^{2}}{\alpha}, \\
& c \approx \frac{1}{z^{3}},
\end{aligned}
$$

where $\alpha$ is thermal diffusivity of material (i.e., 0.53 for concrete).

Note that as part of the limitations of this approach, the radius of the smallest defect that is detectable by thermal image should be around two times larger than the depth in which it is located underneath the surface of the specimen [11].

After employing a heat pulse on the specimen, the temperature decay will follow the following equation [12]:

$$
\Delta T=\frac{Q}{e \sqrt{\pi t}},
$$

where $\Delta T$ is the increase of the temperature on the surface, $Q$ is the quantity of the energy absorbed, and $t$ is considered as time. In the denominator, $e$ is the thermal effusivity which is calculated using the following formula: $e=\sqrt{k \rho C}$, where $k$ is thermal conductivity (for concrete it is $1 \mathrm{Wm}^{-1{ }^{\circ}} \mathrm{C}$ ), $\rho$ is mass density (for concrete it is $2400 \mathrm{kgm}^{-3}$ ), and finally $C$ is specific heat (for concrete it is $800 \mathrm{Jkg}^{-1{ }^{\circ}} \mathrm{C}^{-1}$ ).

For materials of low diffusivity, more uniform heating inside the oven should be used for their preheating process. In such experiments, after the specimen reaches a stable temperature that is higher than the ambient temperature, the specimen will be removed from the oven and during its cooling cycle, the surface temperature will be recorded using an infrared camera. Then the data can be used to verify the previous equation and eventually will help us realize the depth of the defects inside the specimen [13]. In future experiments, the accuracy and the workability of the aforementioned equation for concrete structures will be examined using live thermal imagery.

IRT is a fast and reliable technique. Deployment of the procedure is easy and no direct contact is required. Its oneside deployment makes it favorable for bridges in which we generally have access to only one side of the structure. It is considered a safe technique since there is no harmful radiation involved. A thermal camera and a corresponding software will provide us with a numerical thermal modeling.

On the other hand, it is difficult to manage a uniform heating in active IRT. Thermal contrast is a decaying process and due to this transitory phase, a fast recording IR camera is required. Defects are detectable only if they are located in shallow depths, especially inside concrete pavements. Finally, this system only works when there is a thermal contrast present [12]. In order to overcome these disadvantages, we use image analysis approach to detect the subsurface defects instead of interpreting the raw IR images.

Utilizing the benefits of IR technique, we use IR to fastdetect concrete pavement subsurface defects. A laboratorydesigned experiment was carried out in this study. Thermal transfer modeling was used to assist the IR imaging test setup. In our experiment, we used active thermography but we did not employ live thermal imagery. We used a specific temperature threshold (37 degrees of Celsius) to process our images and get the digital indication of the location of the defects. In this temperature, the amount of thermal energy that is diffused on the surface of the specimen is calculated and deducted from the thermal image using the image processing technique.

\section{Test Setup}

In this research, we used a FLIR thermal camera, model T430sc, to capture image data. Object distance is set at 0 to 1 meter and atmospheric temperature is set at 20 degrees of Celsius (close to lab temperature). Relative humidity is considered around 50 percent and emissivity was set as default, that is, 0.95 . The wavelength range of the camera is between 7 and 14 Microns. Integration time in the camera for our study is 12 milliseconds.

In order to use this method on the concrete and asphalt pavements in the field, it is ideal to maintain a specific temperature and humidity throughout the entire testing procedure; otherwise the changes should be included in the specifications of the camera and they should be mentioned in the results.

This model of camera uses uncooled micro bolometer also known as thermal detector [14]. Thermal sensitivity of the camera is less than 0.045 degrees at around 30 degrees of Celsius with the accuracy of \pm 2 percent.

This camera uses a technique called UltraMax, a type of image enhancement technology, to capture a series of thermal images and combine them into a new image. UltraMax uses the natural movement of the hand to capture an image set in which each image is slightly offset from the others. This gives us a higher number of data compared to a single image. By using this technique, we managed to increase the pixels from 76800 to 307200 , which will provide a better quality of thermal image.

We developed a simple procedure to fabricate lab scale concrete specimen. In order to mimic the real pavement subsurface defects, multiple delamination was introduced in the concrete cylinder. For this test we casted multiple concrete cylinders and inserted different shapes of delamination in different depths of the specimen to realize which depth is the most ideal and gives the best contrast in thermal images. The diameter of each cylinder is 10.16 centimeters ( 4 inches) and the height is 20.32 centimeters ( 8 inches). Defects are 3 by 3 and 4 by 4 square centimeters Styrofoam rectangular cubes and their height varies depending on their depths.

Our camera did not record a visible temperature change for the defects that were buried in more than 3 centimeters deep inside the concrete. On the other hand, we were able to detect defects with the depths in between 1 and 


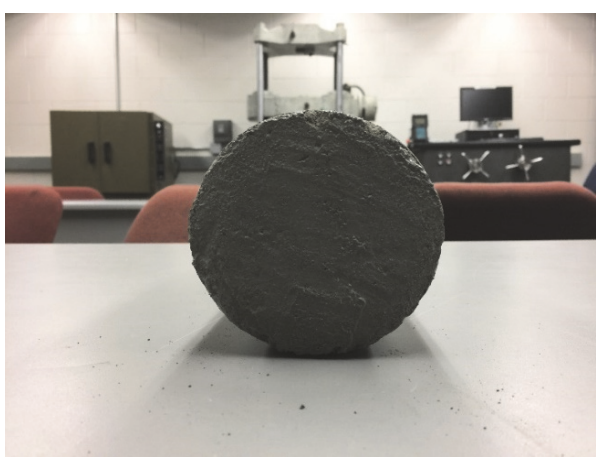

(a)

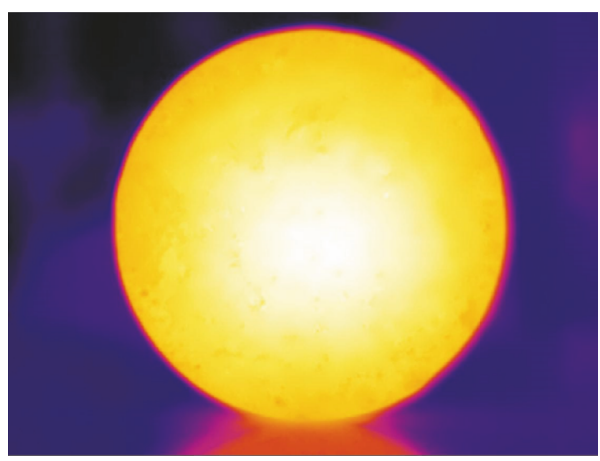

(b)

FIGURE 1: (a) Visual image of the specimen; (b) thermal image of specimen with defects that are inserted in the depth of 2 centimeters (heated up using heat blower).

2.5 centimeters. For the defects with the depth that is less than 1 centimeter, the images were clear enough for further studying.

Active heating input was provided from one end surface of the specimen. This type of heating is common for thermal inspection of specimen and the heat source can be a laser or other types of heat induction devices [15]. In order to simulate the cooling cycle of the specimen, two different types of heating procedures were used to increase the temperature. At first, the specimen was heated up using an oven for ten minutes while keeping the bottom side of the concrete inside iced water. Although this method induced uniform heat on the surface, the contrast in the thermal images was not sharp enough for us to be able to use it for further evaluation. This type of heating procedure will be useful when live temperature inspection is conducted using thermal video.

In the second approach, a hot air blower was used to heat up the surface for 15 to 20 minutes. With this method we got a clearer thermal image and the edge of the defects was more visible. The highest temperature rose up to 63.7 degrees of Celsius. This method of heating loosely simulates the type of ambient heating that is exposed to the bridge deck.

\section{Experimental Results}

3.1. Raw Thermal Images. The visible image of the specimen is shown in Figure 1(a). The thermal image of the same specimen is on display in Figure 1(b). Note that the temperature difference at the bottom of this image, right under the cylinder, is simply the reflection of the heat from the surface of the table. A $4 \mathrm{~cm}$ Styrofoam cube was used as a delamination that is placed at 2 centimeters deep inside the specimen. The surface is heated up to 45 degrees of Celsius. The temperature of the surface that is located on top of the delamination is visibly higher than the sound area that helps us to estimate where the defects are inserted. However, the heat is expanded in a wider area than the actual size of the delamination underneath the surface. As it is already mentioned, the surface on top of the delamination has a higher temperature and since concrete has a low heat conductivity, therefore, the heat will be trapped in the body of the specimen and there will be a temperature difference between the delaminated area in the middle and the edge of the specimen which is more exposed to the surrounding air. Similar method of inspection is used inside industrial plants [16]. Due to this temperature difference, heat transfers from delaminated area towards the boundary that is relatively colder. This is one reason why the area with higher temperature is wider than the area of the delamination itself inside the specimen.

Using the type of the thermal camera that we have for this experiment, we can detect defects clearly under the concrete surface up to the depth of 2 centimeters. Nevertheless, as it will be mentioned in more detail in the next sections, the shallow defects, up to one centimeter, will have the best temperature contrast in the IR thermal images [17].

Figures 2(a) and 2(b) are of the same specimen, which has two defects (both are $3 \mathrm{~cm}$ Styrofoam cubes), inserted in 5 and 10 millimeters underneath the surface. As it is visible, the type of heating procedure is an important factor in terms of having a clearer thermal image. In Figure 2(a), the specimen was heated up inside an oven for 30 minutes. More than 50 images were taken in a time span of 2 hours and none of them had a clear indication of defects that were trapped underneath. The heat transfer was steady all over the surface of the specimen and there was no indication of a noticeable temperature difference to the point that it can be used for further studies.

As for Figure 2(b), a heat blower was used for about 20 minutes to heat up the surface. The thermal image was taken 3 minutes into the cooling cycle of the specimen. The highest temperature was around 63.5 degrees of Celsius. Temperature difference between the delaminated area and the sound area is clearly visible in this image. It also indicates that the depth has a major effect on the temperature gradient that is visible on the surface of delaminated areas of the specimen. The defect that is shallower underneath the surface has more effect on the temperature of the surface of the specimen. This difference will eventually fade away as the time passes during the cooling cycle and there will be less indication of the temperature difference between the two delaminated areas. The image in Figure 2(c) was taken 20 minutes into the cooling cycle and the temperature difference is not clear 


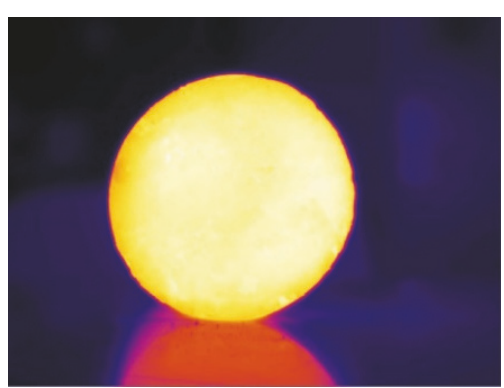

(a)

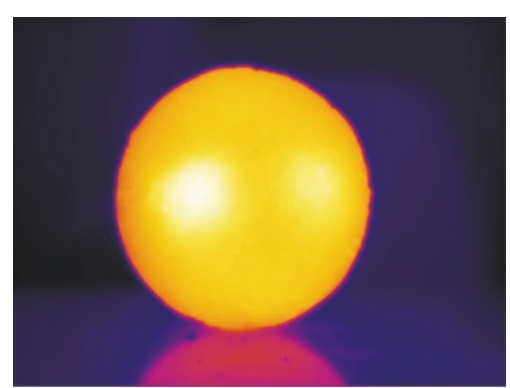

(b)

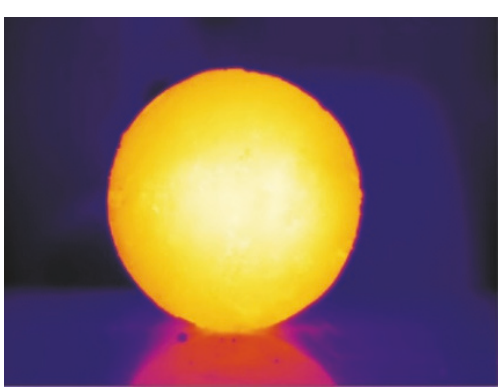

(c)

FIgURE 2: (a) Specimen with defects that are inserted in the depths of 5 and 10 millimeters (heated up using an oven), (b) specimen with defects that are inserted in the depth of 5 and 10 millimeters (heated up using heat blower), image taken 2 minutes into the cooling cycle, and (c) specimen with defects that are inserted in the depth of 5 and 10 millimeters (heated up using heat blower), image taken 20 minutes into the cooling cycle.

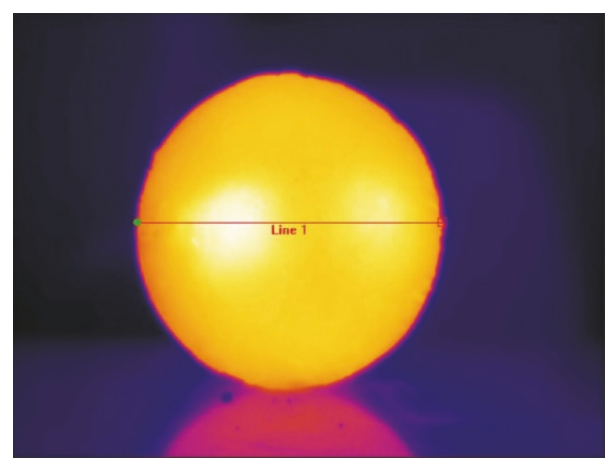

FIGURE 3: Line 1 is used to plot a temperature related graph of the surface of the specimen.

enough; therefore we cannot use a longer time span to take our images from the surface.

\subsection{Interpretation of Temperature versus Distances' Graph. A} graph is plotted using the temperatures that are assigned to each pixel on line 1 (shown in Figure 3 ) that is put in the middle of the surface of the specimen. This line intersects the areas with the most temperature gradient inside the IR thermal image. By using this line, we can get the temperature of each pixel located on these areas.

As is shown inside the graph (Figure 4), the location of each defect is visible with a very good accuracy. It is also clearly visible that there is a depth difference between the two defects by judging the temperature difference on $y$-axis. The pattern inside this graph shows a clear connection between the depth in which the defect is located underneath the surface and the amount of temperature that is reflected from the top of the surface. In the graph, we can clearly see that there is 2 degrees of Celsius temperature gap between defect 1 (61.5 degrees of Celsius) and defect 2 (63.5 degrees of Celsius).

In live imagery, a similar graph can be used in order to get the temperature gradients during the heating or cooling cycles. The information can later on be used to both locate the defects and, if possible, calculate the depth of each defect inside the concrete pavement. The relation between the depth

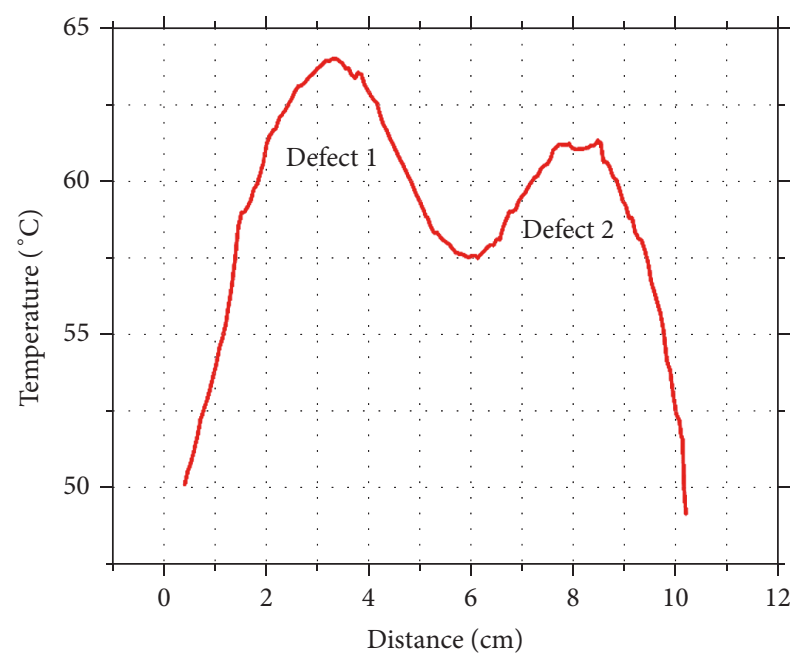

Figure 4: Temperature versus distance graph assigned to line 1 in Figure 3.

and the observation time is already mentioned in active and passive IRT section.

3.3. Image Processing Data. As is mentioned in the previous sections, the image for the specimen with two defects inserted in 5 and 10 millimeters' depth underneath the surface is used for image processing purposes. For defects that are under 1 centimeter inside the concrete pavement, the thermal image presents the clear data with best contrast that enables us to determine the edges of the delaminated area with higher temperature. To refine the difference between each temperature set, we are going to use a different filter that magnifies the temperature contrasts. This filter only manipulates the coloring assigned to each temperature pixel that results in a sharper and clearer temperature contrast inside the image (Figure 5).

The minimum temperature in this image is around 21 degrees of Celsius and the maximum temperature is around 63.5 degrees of Celsius. In the processed image, we used 255 different thresholds, known as gray thresholds, and each 
TABLE 1: Area comparison.

\begin{tabular}{lccc}
\hline & Number of pixels & IR measurement of area & Percentage of accuracy (POA) \\
\hline Actual area of the defects inside the specimen & 30276 & $9 \mathrm{~cm}^{2}$ & $100 \%$ \\
Area of processed image of the $5 \mathrm{~mm}$ deep defect & 21286 & $6.33 \mathrm{~cm}^{2}$ & $\frac{6.33}{9} \times 100=70.33 \%$ \\
Area of processed image of the $10 \mathrm{~mm}$ deep defect & 5727 & $1.7 \mathrm{~cm}^{2}$ & $\frac{1.7}{9} \times 100=18.88 \%$ \\
\hline
\end{tabular}

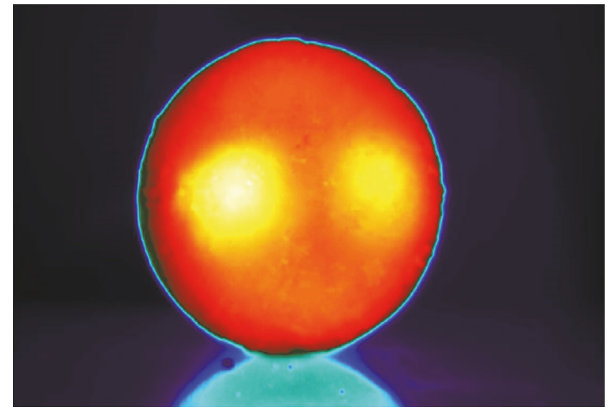

Figure 5: Thermal image after application of color filter.

of these thresholds is assigned to a specific temperature. A gray threshold of 226 which corresponds to 37 degrees of Celsius (Figure 6(a)) is used to get the best contrast between the delaminated area and the sound area. By choosing the threshold, we are eliminating all the pixels that have a temperature more than a certain degree of Celsius [18]. This helps us to clarify the area of the delaminated surface for further study inside the computer [19]. In Figure 6(b), the actual areas of the defects are added to the processed image. This can give us a sense of the difference between the actual area and the area that the thermal camera can detect.

As is clearly shown in the processed images, for shallower delamination, the area with higher temperature on the surface will be closer to the actual area of the surface of the delamination [20]. It is also worth mentioning that, in the shallower defect, the heated area exceeds the actual area on the left side. This can be due to the heat diffusion that is caused by the temperature difference between the boundary of the cylinder and the middle part of it where the defects are located.

The data that we got from the image processing enables us to get more information from the raw images using computer coding. Through this information, we can extrapolate mathematically accurate data to help us identify the characteristics of the delaminated areas. Table 1 contains an area comparison between the delaminated areas that are shown in the processed thermal image and the actual area of the surface of the defects inserted in the concrete specimen.

Using these data, we came up with a quantitative measuring method that helps us calculate the percentage of accuracy (POA) of the processed thermal image. We found the percentage of difference between the actual area of the delamination and the area that is shown in the processed image. Using POA, we can come up with a system that enables us to evaluate the area of an unknown delamination

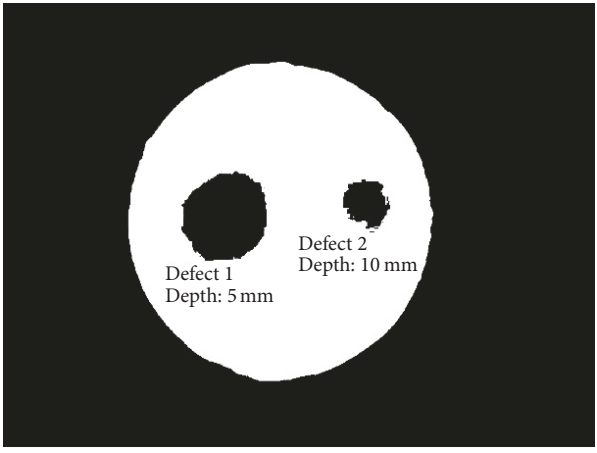

(a)

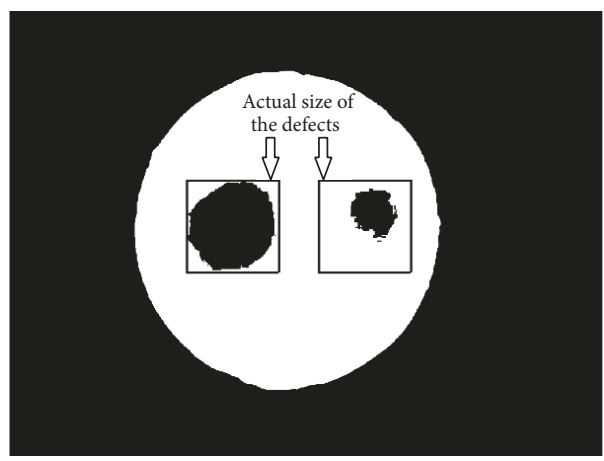

(b)

Figure 6: (a) Processed image using a 37 degrees of Celsius threshold. (b) A comparison between the actual size of the delamination and its size in the processed image.

in accordance with its depth underneath the surface of the concrete specimen. We should note that the temperature threshold that we use is the main factor in determining POA; that is, if we choose a bigger threshold we may end up having entirely different POA. In some cases, when the threshold is bigger than a certain number, the delaminated area in the processed image will overlap the actual size of the delamination.

Since we know the actual diameter of the cylinder, in order to calibrate the processed image, we drew a line from either end of the cylinder borders. This line occupies 587.82 pixels in the processed image and since we know that the diameter of the cylinder is 10.16 centimeters, the number of pixels in each centimeter can easily be calculated which is 58 pixels for each centimeter of the image. Therefore, there are 3364 pixels in every square centimeter of the image. Using this number, we came up with Table 1 for our area comparison. 


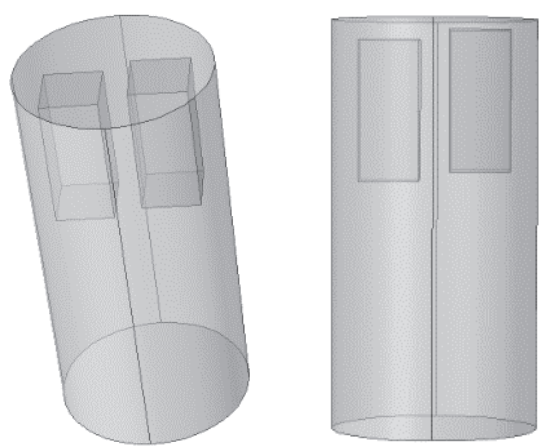

FIgURE 7: Defects location in the software model.

According to the data that is extrapolated from the processed image, the delamination that is located in the depth of 5 millimeters inside the concrete pavement has a POA of more than 70 percent with respect to the actual size. The POA drastically decreases when the delamination is 5 millimeters deeper. This clearly shows that the accuracy of the thermal image is strongly related to the depth of the delamination underneath the surface.

3.4. Modeling the IR Test. Heat transfer in solids is simulated inside the computer to further understand the IR test setup [21]. Concrete is the main material and Styrofoam cubes are used for the defects underneath the surface. A heat source of 300 watts per meter cube is applied on top of the concrete in a uniformed pattern and the bottom side was kept at 0 degrees of Celsius in order to see the heat transfer inside the model (Figures 7 and 8). The defects are of the same size as the defects in our experimental model and they are put in the same exact depths as well.

As it is shown in Figure 8, a guideline is put on top of the model that covers the surface on top of the defects and their surrounding sound areas. This line was used to extrapolate temperature-distance graph from the model. This will help us to detect the defects underneath the model as we did in the actual experimental test and it helps us to learn about the depth difference between the two delaminations by using the data that is shown inside the graph.

In the software model, temperature contours show all the areas with the same unique temperature compared to their surrounding area [22]. Since our heating process in the experimental test was not uniform, the heated areas are different in the software results from the actual experimental results especially on the deeper defected area.

This graph from the model (Figure 9) follows the same pattern that we got from experimental data. From this graph, the same as the experimental graph, we can come up with a pattern that gives a relation between the temperature difference and the depth in which the defects are located.

3.5. Integration of Simulation and Experimental Result. Judging the temperature graphs extrapolated from both experimental data and the data from the software, we realize that both graphs follow the same pattern. The maximum temperature, which is located on top of the shallower delamination, is almost the same in both graphs with a magnitude close to 63.5 degrees of Celsius in experimental graph and 62.5 degrees of Celsius in the simulation graph. On the other hand, there is a temperature difference between the simulation and experimental data when it comes to the temperature on top of deeper delamination. This can be because in the software we applied a totally uniformed heat flux to the top surface of the cylinder but in reality our heating process, using a heat blower, was not performed in a uniformed manner.

In future experiments and in order to get closer results to what we get from the software we can use more uniformed ways of inducing heat such as putting the specimen on a hot surface for a certain amount of time and then take thermal images from it. In Figure 10, a distance versus temperature graph for both simulation and experimental data is shown for comparison. In this graph, we can clearly see that our simulation and experimental data are following the same pattern when it comes to the relationship between the depth of defects and the temperature of the surface of the specimen.

Since we were able to confirm the pattern between the depth of the delamination and the temperature on the surface of the model in both experimental test and the software, in future studies we can use this pattern in the software and predict the results that we will get from experimental tests. This will help us to optimize our experiments inside the software before we start the process inside the lab.

\section{Conclusion}

In this research, an experimental test has been conducted on a concrete specimen with two defects located in different depth. One defect is located at 5 millimeters and the other one is located at around 10 millimeters underneath the surface of the specimen. After inducing heat on the surface of the specimen for a certain amount of time, thermal images have been taken and then used for further studies.

A temperature versus distance graph is drawn using the data that we got from thermal image. A clear pattern has been found between the temperature gradient and the depths of the defects underneath the surface. There is 2 degrees of Celsius temperature difference between the surfaces on top of the two defects. As it is shown inside the graph, deeper defects trapped less heat on top of it; therefore the camera read less temperature from the top surface of the deeper defect compared to the shallower defect.

A simulated model of the same test has been conducted inside the computer and the results have been compared with the ones that were extrapolated from the experimental test. The comparison showed us a clear link between the experimental and software results. We also have witnessed the same pattern in the graph that we got from the simulation.

The same thermal image then was used for image processing purposes and the difference between the areas of the processed image of the defects and the actual size of the defects has been found. The area of the defect that is placed in 5 -millimeter depth inside of the specimen is about $6.33 \mathrm{~cm}^{2}$ which is 30 percent less than the actual size of the specimen which is $9 \mathrm{~cm}^{2}$. The size of the defect that was placed in $10-$ millimeter depth inside of the specimen is around $1.7 \mathrm{~cm}^{2}$ that 

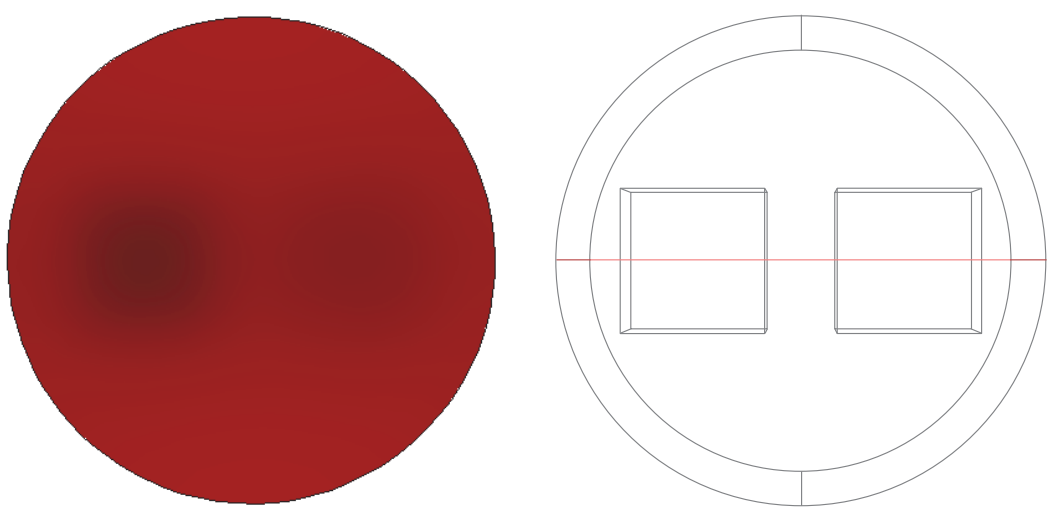

FIGURE 8: Line that is used to plot a temperature related graph of the surface of specimen.

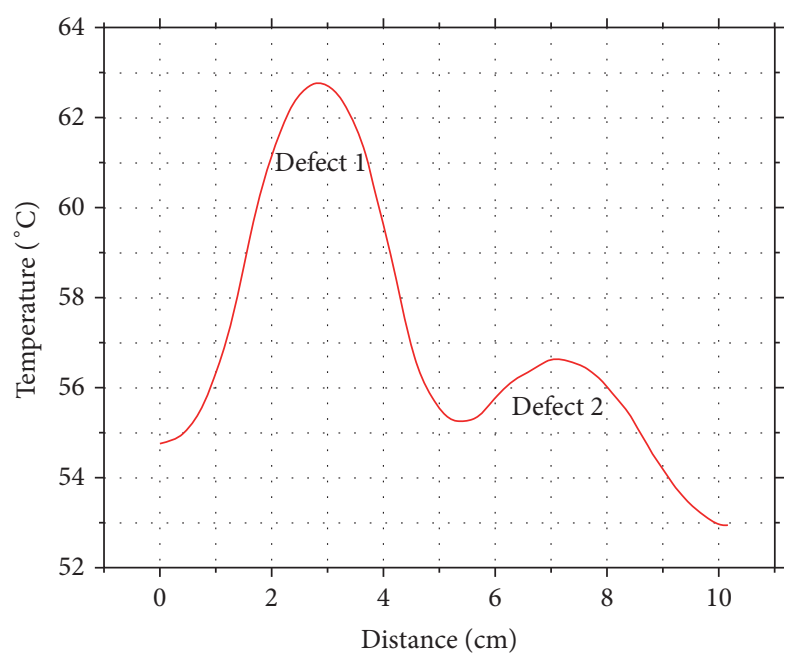

FIGURE 9: Temperature versus length graph assigned to the line in Figure 8.

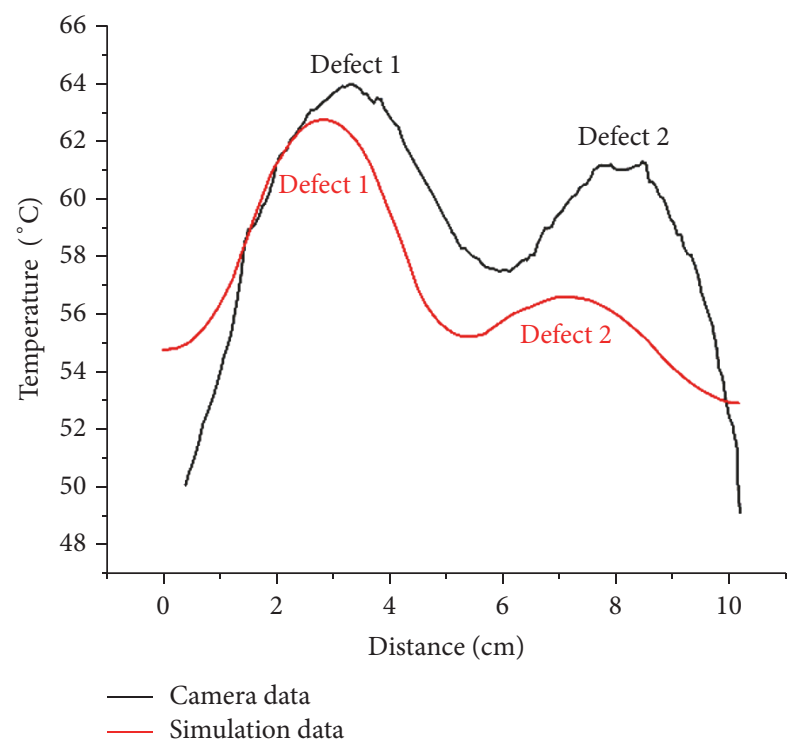

FIGURE 10: Comparison between the location of defects on both camera and software graphs. is around 80 percent less than the actual size of the defect. By using these data, we could come up with a system to figure out the accuracy of the results in thermal images which we called percentage of the accuracy (POA). As the depth of the defect inside the specimen increases, the amount of the POA decreases drastically.

The heat transfer simulation results provide us with a good method to refine our experimental settings. With a set of accurate material parameters, we are able to predict the experimental tests under a controlled laboratory environment. The proposed IRT method has been validated to be a good alternative to inspect concrete pavements.

\section{Conflicts of Interest}

The authors declare that they have no conflicts of interest.

\section{References}

[1] D. J. Titman, "Applications of thermography in non-destructive testing of structures," NDT and E International, vol. 34, no. 2, pp. 149-154, 2001.

[2] ASTM, "Standard Test Method for Detecting Delamination in Bridge Decks Using Infrared Thermography," in Proceedings of the ASTM Designation D4788-03 ed. ASTM International, West Conshohocken, Penn, USA, 2014.

[3] S. Hiasa, R. Birgul, and F. N. Catbas, "Infrared thermography for civil structural assessment: demonstrations with laboratory and field studies," Journal of Civil Structural Health Monitoring, vol. 6, no. 3, pp. 619-636, 2016.

[4] A. Watase, R. Birgul, S. Hiasa, M. Matsumoto, K. Mitani, and F. N. Catbas, "Practical identification of favorable time windows for infrared thermography for concrete bridge evaluation," Construction and Building Materials, vol. 101, pp. 1016-1030, 2015.

[5] M. R. Clark, D. M. McCann, and M. C. Forde, "Application of infrared thermography to the non-destructive testing of concrete and masonry bridges," in Proceedings of the Application of Infrared Thermography to the Non-destructive Testing of Concrete and Masonry Bridges, pp. 265-275, 2003.

[6] R. B. Mignogna, R. E. Green Jr., J. C. Duke Jr., E. G. Henneke II, and K. L. Reifsnider, "Thermographic investigation of highpower ultrasonic heating in materials," Ultrasonics, vol. 19, no. 4, pp. 159-IN2, 1981. 
[7] J. Roche, D. Balageas, B. Lamboul et al., "Passive and active thermography for in situ damage monitoring in woven composites during mechanical testing," in Proceedings of the Review of Progress in Quantitative Nondestructive Evaluation, vol. 32, pp. 555-562, Denver, Col, USA, 2013.

[8] R. Richter, C. Maierhofer, and M. Kreutzbruck, "Numerical method of active thermography for the reconstruction of back wall geometry," NDT and E International, vol. 54, pp. 189-197, 2013.

[9] P. Cielo, X. Maldague, A. A. Deom, and R. Lewak, “Thermographic nondestructive evaluation of industrial materials and structures," Materials Evaluation, vol. 45, no. 4, pp. 452-460, 1987.

[10] J. J. Allport and S. L. McHugh, "Quantitative evaluation of transient video thermography," in Review of Progress in Quantitative Non-Destructive Evaluation, D. O. Thompson and D. E. Chimenti, Eds., pp. 253-262, 1988.

[11] V. P. Vavilov and R. Taylor, "Theoretical and practical aspects of the thermal non-destructive testing of bonded structures," Research Techniques in NDT, pp. 239-279, 1982.

[12] X. P. V. Maldague, Theory and Practice of Infrared Technology for Nondestructive Testing, John Wiley and Sons, 2001.

[13] D. Wu, A. Salerno, B. Schönbach, H. Hallin, and G. Busse, "Phase-sensitive modulation thermography and its applications for NDE," in Proceedings of the Thermosense XIX: An International Conference on Thermal Sensing and Imaging Diagnostic Applications, pp. 176-183, usa, April 1997.

[14] P. W. Kruse, Uncooled Thermal Imaging: Arrays, Systems and Applications, Spie Press, 2001.

[15] R. Osiander, J. W. M. Spicer, and J. M. Amos, "Thermal inspection of $\mathrm{SiC} / \mathrm{SiC}$ ceramic matrix composites," in Proceedings of the Thermosense XX, pp. 339-349, usa, April 1998.

[16] S. Harishkumar, V. R. Mohammed, and B. M. Mujtaba, "Detection of hot spots by thermal imaging to protect power equipments," International Journal of Students' Research Technology \& Management, pp. 64-66, 2014.

[17] M. Vollmer and K. Möllmann, Infrared Thermal Imaging: Fundamentals, Research and Applications, John Wiley \& Sons, 2011.

[18] C. Ibarra-Castanedo, D. González, M. Klein, M. Pilla, S. Vallerand, and X. Maldague, "Infrared image processing and data analysis," Infrared Physics \& Technology, pp. 75-83, 2004.

[19] J. C. Russ and F. B. Neal, The Image Processing Handbook, CRC Press, Boca Raton, Fla, USA, 5th edition, 2002.

[20] N. Rajic, "Principal component thermography for flaw contrast enhancement and flaw depth characterisation in composite structures," Composite Structures, vol. 58, no. 4, pp. 521-528, 2002.

[21] S. Carcangiu, B. Cannas, G. Concu, and N. Trulli, "Modeling of active infrared thermography for defect detection in concrete structures," in Proceedings of the COMSOL Conference, pp. 1-7, 2012.

[22] V. Gerlich, K. Sulovská, and M. Zálešák, "COMSOL Multiphysics validation as simulation software for heat transfer calculation in buildings: Building simulation software validation," Measurement: Journal of the International Measurement Confederation, vol. 46, no. 6, pp. 2003-2012, 2013. 

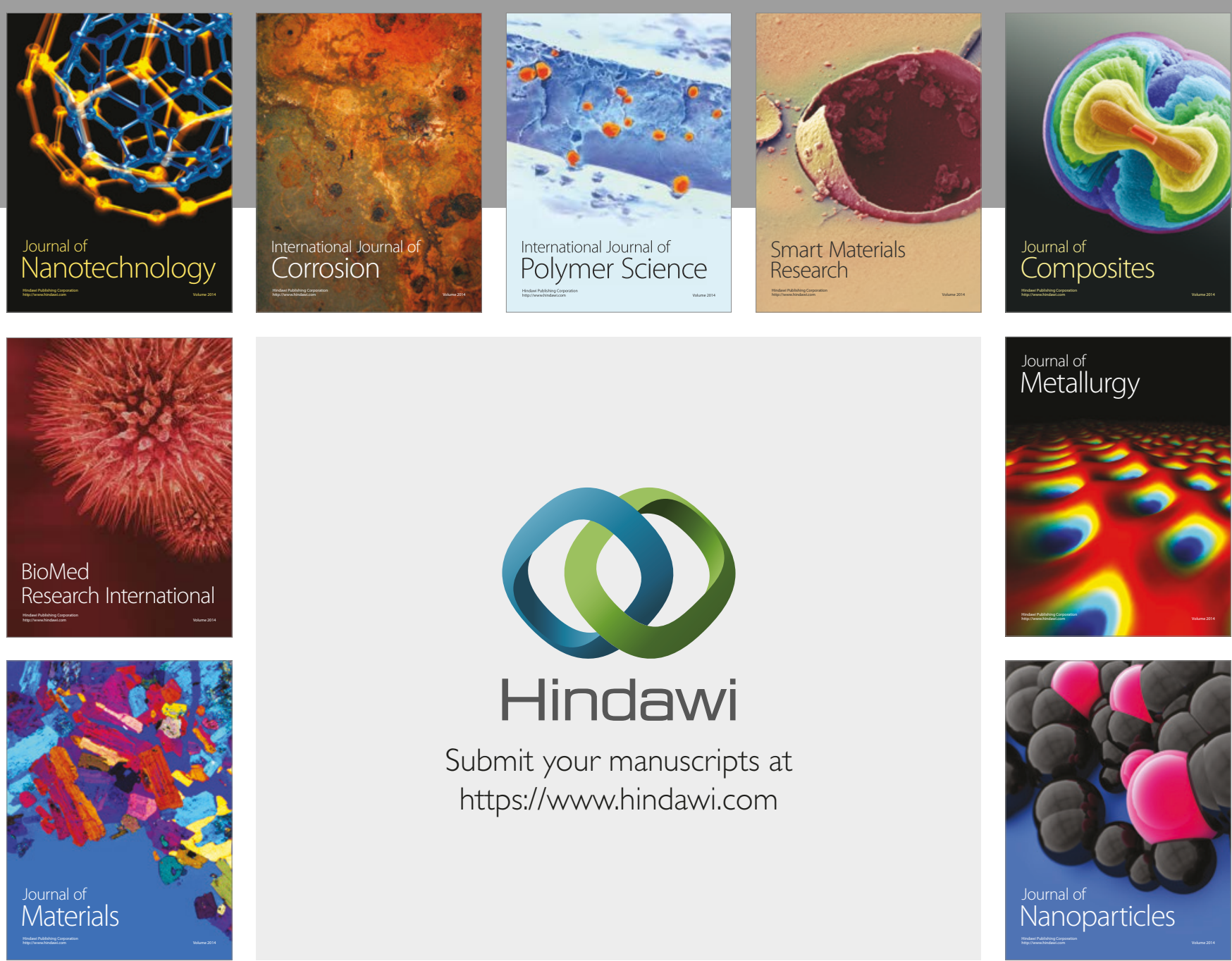

\section{Hindawi}

Submit your manuscripts at

https://www.hindawi.com
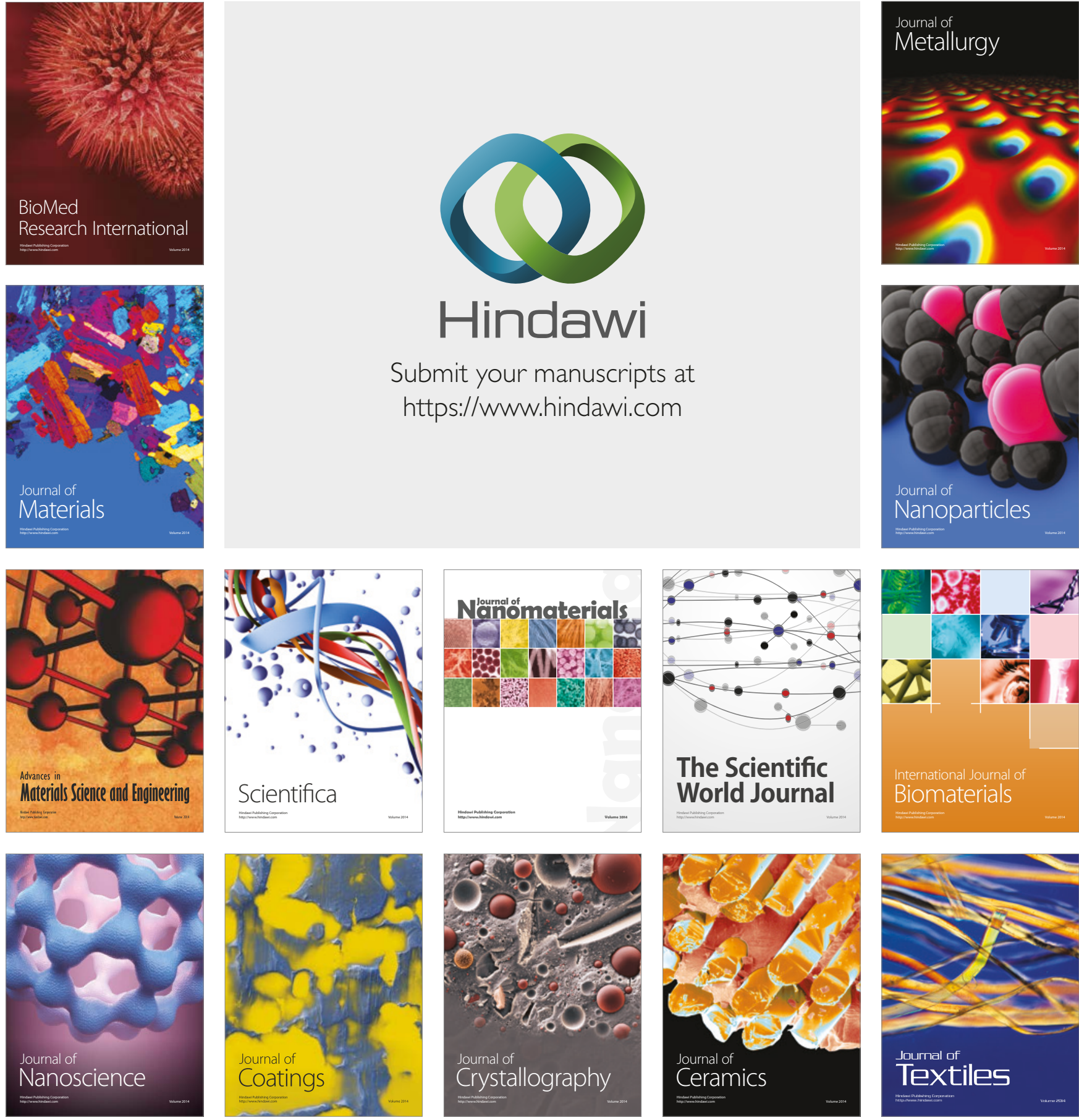

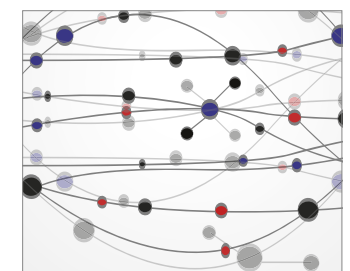

The Scientific World Journal
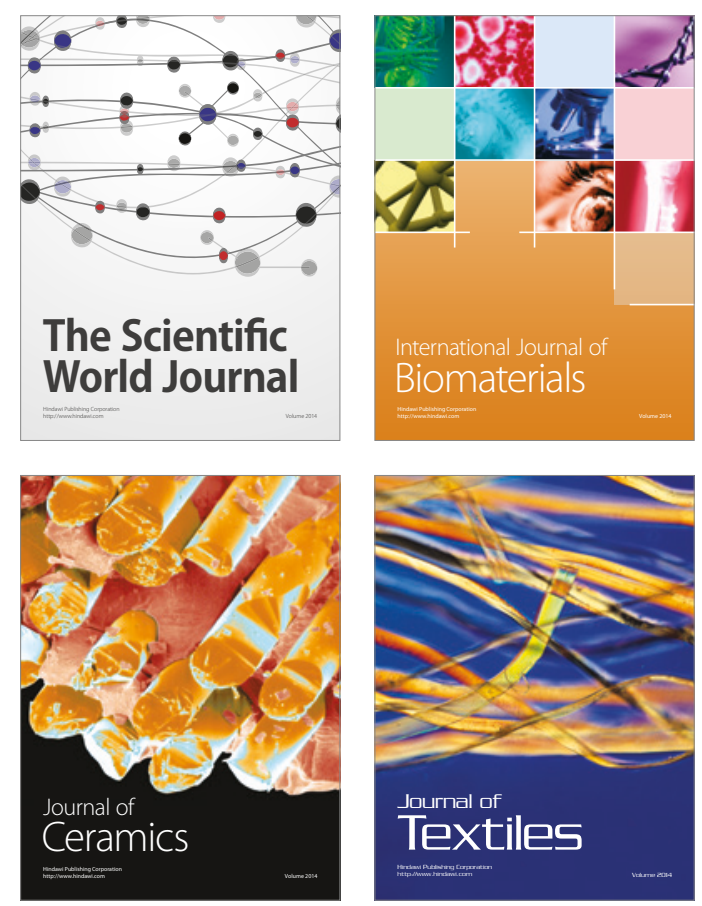